\title{
Neural stem cells and neuro/gliogenesis in the central nervous system: understanding the structural and functional plasticity of the developing, mature, and diseased brain
}

\author{
Masahiro Yamaguchi ${ }^{1}$ (1) Tatsunori Seki ${ }^{2} \cdot$ Itaru Imayoshi $^{3} \cdot$ Nobuaki Tamamaki $^{4}$. \\ Yoshitaka Hayashi $^{5}$ - Yoshitaka Tatebayashi ${ }^{6} \cdot$ Seiji Hitoshi $^{5}$
}

Received: 9 October 2015/Accepted: 18 October 2015/Published online: 17 November 2015

(C) The Physiological Society of Japan and Springer Japan 2015

\begin{abstract}
Neurons and glia in the central nervous system (CNS) originate from neural stem cells (NSCs). Knowledge of the mechanisms of neuro/gliogenesis from NSCs is fundamental to our understanding of how complex brain architecture and function develop. NSCs are present not only in the developing brain but also in the mature brain in adults. Adult neurogenesis likely provides remarkable plasticity to the mature brain. In addition, recent progress in basic research in mental disorders suggests an etiological link with impaired neuro/gliogenesis in particular brain regions. Here, we review the recent progress and discuss future directions in stem cell and neuro/gliogenesis biology by introducing several topics presented at a joint meeting of the Japanese Association of Anatomists and the Physiological Society of Japan in 2015. Collectively, these topics indicated that neuro/gliogenesis from NSCs is a common event occurring in many brain regions at various
\end{abstract}

Masahiro Yamaguchi

yamaguti@m.u-tokyo.ac.jp

$\triangle$ Tatsunori Seki

sekit@tokyo-med.ac.jp

1 Department of Physiology, Graduate School of Medicine, The University of Tokyo, Tokyo, Japan

2 Department of Histology and Neuroanatomy, Tokyo Medical University, Tokyo, Japan

3 The Hakubi Center, Kyoto University, Kyoto, Japan

4 Department of Morphological Neural Science, Graduate School of Medical Sciences, Kumamoto University, Kumamoto, Japan

5 Department of Integrative Physiology, Shiga University of Medical Science, Shiga, Japan

6 Affective Disorders Research Project, Tokyo Metropolitan Institute of Medical Science, Tokyo, Japan ages in animals. Given that significant structural and functional changes in cells and neural networks are accompanied by neuro/gliogenesis from NSCs and the integration of newly generated cells into the network, stem cell and neuro/gliogenesis biology provides a good platform from which to develop an integrated understanding of the structural and functional plasticity that underlies the development of the CNS, its remodeling in adulthood, and the recovery from diseases that affect it.

Keywords Neural stem cells · Neurogenesis · Gliogenesis · Plasticity

\section{Introduction}

The brain is a highly complex organ that consists of various types of neurons and glia. How the complex brain architecture develops from neural stem cells (NSCs) is one of the central questions of neuroscience. Much interest has been paid to the mechanisms of NSC maintenance, proliferation, migration, and differentiation into various cell types [1]. Recent advances in molecular and developmental biology have strongly facilitated this understanding.

A second focus of interest in neurogenesis is the fact that neurogenesis continues throughout life in particular brain regions. The long-held belief that neurons are no longer regenerated once development of the central nervous system (CNS) ceases was challenged about 50 years ago [2]. Progress in the several decades since has made adult neurogenesis in the hippocampus and olfactory system widely accepted [3]. The mechanisms underlying adult neurogenesis and its functional significance have been pursued as an important focus of neurogenesis research. 
Further, the finding that newly generated neurons and glia from NSCs can be used in the treatment of degenerative disease and trauma of the CNS has attracted the wide interest of neuroscientists and clinicians in stem cell biology. These therapeutic approaches are strongly facilitated by recent advances in ES cells and iPS cells [4]. In addition, further interest has been shown in the correlation between impaired neuro/gliogenesis and psychiatric diseases, including mood disorders and chronic stress syndromes [5], along with recent progress in basic research into these diseased states. A closer understanding of the nature of NSCs and neuro/gliogenesis is therefore crucial to various aspects of neuroscience, including the development, remodeling, and restoration of brain architecture and function.

Stem cell and neuro/gliogenesis biology is also strongly linked to the plastic potential of the CNS. NSCs show remarkable plasticity in differentiating into a variety of cell types that play a critical role in the formation of the complex brain architecture. After the brain develops to maturity, its plastic function is generally exhibited through the plasticity of preexisting neurons generated during the developmental and neonatal periods. It is notable, however, that NSCs in the mature brain likely confer far stronger plastic potential. In addition, restoring appropriate neuro/ gliogenesis in the diseased brain can be regarded as the process of adjusting the brain's plasticity for structural and functional restoration.

Thus, stem cell and neuro/gliogenesis biology highlights the remarkable plastic potential of the brain. This research field provides an opportunity to integrate structural plasticity and functional plasticity at the cellular, circuit and behavioral levels and has attracted wide interest from basic scientists, clinicians, and society. In 2015, the Japanese Association of Anatomists and the Physiological Society of Japan held a joint annual meeting in Kobe, Japan. One symposium, "Neurogenesis from embryo to adult," attracted a large audience and generated vigorous discussion. In this review article, we summarize the five presentations we made in this symposium, with the goal of providing a comprehensive understanding of the current progress and future directions of this research field.

We briefly introduce the individual topics in the symposium and then go into detail. First, Itaru Imayoshi introduced the role of transcription factors in the self-renewal and differentiation of NSCs in the developing neuroepithelium. Dr. Imayoshi revealed the pivotal role of the expression dynamics of basic helix-loop-helix (bHLH) transcription factors, oscillatory or sustained, on the NSC self-renewal and fate decision. Second, Tatsunori Seki raised the question of how NSCs produce granule cells in the embryonic and early postnatal hippocampus and how the early neurogenesis is succeeded by adult neurogenesis.
Dr. Seki revealed that the first progenitors of granule cells arising in the dentate anlage in the developing hippocampus express glial fibrilar acidic protein (GFAP) and that these GFAP-expressing progenitors form a proliferative zone in the subpial and hilar zones during the perinatal stage and later in the subgranular zone, where neurogenesis continues throughout life. Third, Nobuaki Tamamaki introduced the emerging view that adult neurogenesis may also occur in the neocortex. Dr. Tamamaki revealed that neuronal progenitors can be induced in the leptomeninges of the neocortex by electrical stimulation and showed their migration and differentiation in the neocortex. Fourth, Masahiro Yamaguchi introduced how adult-born olfactory neurons are selected for survival or death in the olfactory bulb. Dr. Yamaguchi revealed that the key mechanism of activity-dependent selection is the integration of sensory inputs from the periphery and top-down inputs from higher brain regions. Lastly, Yoshitaka Hayashi, Yoshitaka Tatebayashi, and Seiji Hitoshi introduced the relation between major depressive disorder and oligodendrocyte genesis in the human prefrontal cortex. Hayashi et al. established a method to quantify oligodendrocytes and their progenitors and revealed the dysfunction of oligodendrocyte development in the diseased prefrontal cortex.

\section{Oscillatory expression of basic helix-loop-helix transcription factors in NSCs}

NSCs are multipotent and self-renewable cells that give rise to neurons, astrocytes, and oligodendrocytes in the brain [6]. NSCs of the lateral ventricular wall in the forebrain undergo changes in morphology and produce different progeny as brain development proceeds. NSCs begin as neuroepithelial cells, become radial glial cells, and then finally develop many astrocytic characteristics in the adult brain [7].

During neural development, NSCs (neuroepithelial cells/radial glial cells) initially undergo symmetric cell division: each NSC divides into two NSCs. By repeating symmetric cell division, NSCs proliferate extensively. These cells then undergo asymmetric cell division: each NSC divides into two distinct cell types, one NSC and one immature neuron/neuronal precursor or a basal progenitor cell. Immature neurons or neuronal precursor cells migrate outside the ventricular zone (VZ) into the outer layers, where they become mature neurons, whereas basal progenitor cells migrate into the subventricular zone (SVZ), proliferate further, and give rise to more neurons. By repeating asymmetric cell division, NSCs sequentially give rise to distinct types of neurons or basal progenitor cells. After the production of neurons, NSCs finally differentiate into glial cells, but some of them are maintained as NSCs in the postnatal and adult brain. 
bHLH transcription factors play pivotal roles in the selfrenewal of NSCs and fate determination of neurons, astrocytes, and oligodendrocytes [8-13]. Repressor bHLH factors such as Hes1 regulate the self-renewal of NSCs as downstream effectors of Notch signaling, whereas proneural bHLH factors such as Ascl1 (also called Mash1) promote neuronal differentiation. Another bHLH factor, Olig2, regulates oligodendrocyte differentiation. However, in addition to Hes1, some bHLH fate determination factors, such as Ascl1 and Olig2, have roles in NSC maintenance or proliferation. In addition, Hes 1 induces astrocyte formation at later stages. It is not completely understood how these various and sometimes opposing functions of each bHLH factor in the self-renewal and fate-choice events of NSCs are achieved. Shimojo et al. showed that transcription of some bHLH genes is differentially regulated in NSCs and differentiating cells [14], prompting Imayoshi et al. to analyze the expression dynamics of bHLH factors in further detail [15].

The imaging results of Imayoshi et al. [15] indicated that bHLH factors are expressed by NSCs in an oscillatory manner and that one of them becomes dominant during the fate choice event (Fig. 1). They then proposed that the multipotent state of NSCs correlates with the oscillatory expression of several bHLH factors, whereas the differentiated state correlates with the sustained expression of a single bHLH factor [8, 9, 15].

To examine the functional significance of these bHLH factor expression dynamics, a novel optogenetic method (photo-activatable Gal4/UAS system) was developed to artificially manipulate the expression patterns of bHLH factors with blue light illumination. This analysis

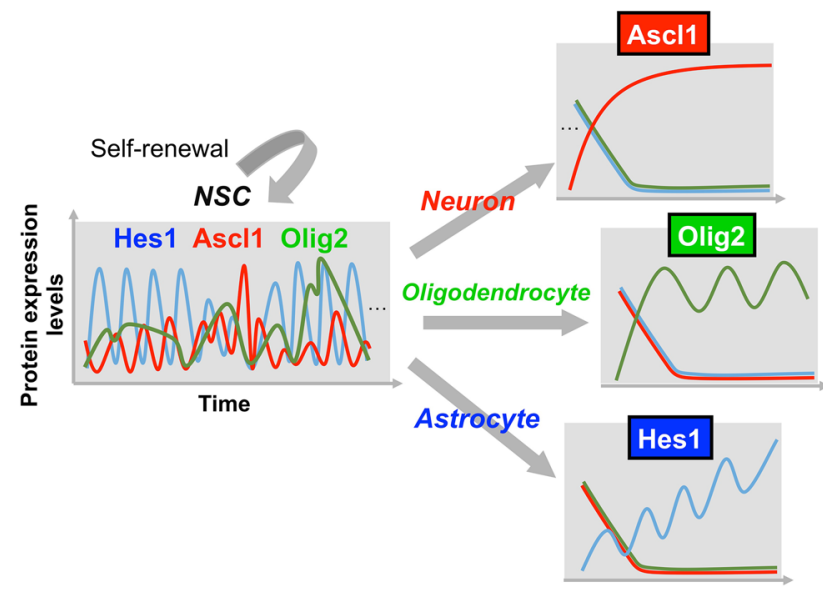

Fig. 1 Expression dynamics of bHLH factors in multipotency and cell fate choice. In multipotent NSCs, levels of Hes1 and Ascl1 oscillate with periods of $2-3 \mathrm{~h}$, while that of Olig2 oscillates with a period of 5-8 h. By contrast, during cell fate choice, one of the bHLH factors is expressed in a sustained manner, while the others are repressed demonstrated that sustained expression of Ascl1 induces neuronal differentiation, whereas oscillatory expression of Ascl1 activates cell proliferation of NSCs [15]. Thus, a single bHLH factor has contradictory functions depending on its expression dynamics. Imayoshi et al. thus highlighted the importance of the expression dynamics of bHLH factors in the self-renewal, multipotency, and fate determination of NSCs [8, 9, 15] (Fig. 1).

\section{From embryonic to adult neurogenesis in the hippocampus}

During the embryonic period, neurons of both the neocortex and hippocampus are initially generated from the VZ of the pallium: the neocortex develops from the dorsal and lateral pallium and the hippocampus from the medial pallium. In the neocortex, neurogenesis occurs only during the embryonic and early postnatal stages and ceases by the early postnatal stage, as typically occurs in most brain regions. However, neurons are exceptionally produced in the adult hippocampus, where dentate granule cells arise from GFAP-expressing astrocyte-like adult neural progenitor cells, which characteristically differ from embryonic neural progenitor cells [2, 16-18].

In an attempt to understand how neurogenesis persists in the hippocampus, Seki et al. [19] explored the neurogenic process of dentate granule cells from the embryonic to late postnatal stages with special reference to GFAP-expressing progenitors, namely when these progenitors appear during the embryonic period and how they form the granule cell layer. Analysis using Gfap-GFP transgenic mice showed that a distinct Gfap-GFP-expressing cell population first appears in the VZ of the medial pallium around a ventricular indentation or in the dentate notch at the dorsal edge of the fimbria at E13.5-14.5. These are radially oriented cells with apical and/or basal processes that seem to migrate from the $\mathrm{VZ}$ to the marginal zone, a region below the pia surface. No Gfap-GFP expression is found in the dorsal or lateral pallium, which develops into the neocortex.

During the perinatal period, Gfap-GFP cells increase in number and form a migratory stream in the suprafimbrial region, as well as in the subpial region between the fimbria and dentate gyrus, alternatively called the fimbrio-dentate junction [20] or fimbrio-dentate juncture [21]. Three proliferative zones are formed by Gfap-GFP-expressing cells in these regions: the primary dentate matrix in the $\mathrm{VZ}$, secondary dentate matrix in the suprafimbrial area and regions below the pia membrane and hippocampal fissure, and the tertiary dentate matrix in the core region of the dentate gyrus, the putative hilus. Gfap-GFP cells in the migratory stream and the developing dentate gyrus are 
positive for Sox 2 and Ki67, suggesting that they contain proliferative progenitors. They also express Neurogenin2 in the VZ, Tbr2 and NeuroD in the migratory stream and developing dentate gyrus, and Prox1 in the developing dentate gyrus. It is therefore concluded that distinctive GFAP-expressing progenitors arising around the dentate notch form germinal regions in the migratory stream and developing dentate gyrus and gradually differentiate into granule neurons. This also indicates that in the dentate gyrus, astrocyte-like neural progenitors continue to generate granule neurons from the beginning of development and throughout life.

The site of origin, proliferative activity, and migratory pattern of the Gfap-GFP-expressing cells agree well with those suggested by studies with ${ }^{3} \mathrm{H}$-thymidine autoradiography [22], and nestin-GFP [20]. However, these reports did not show the astrocytic features of dentate progenitor cells. Although previous reports showed the existence of GFAP-expressing cells and fibers in the embryonic hippocampus $[21,23,24]$, these are thought to belong to the radial type of astrocytes that function as scaffolds for migrating neuroblasts. However, the existence of abundant Gfap-GFP-expressing cells with neuronal marker proteins clearly demonstrates that principally GFAP-expressing cells and fibers are not derived from radial astrocytes, but rather GFAP-expressing migrating spindle-shaped neuronal progenitors and precursors. This may also suggest the presence of radial glia-independent migration.

Interestingly, the dentate gyrus shares some similarities with the cerebellum in terms of their morphogenesis [18]. For example, the hippocampus and cerebellum are derived from neuroepithelium around the edge (cortical hem and rhombic lip) of the dorsal portions of the telencephalon and metencephalon, respectively, both of which are attached to the choroid plexus (Fig. 2). Both progenitors are generated in the ventricular zone (primary proliferative zone) and migrate to the subpial region, in which secondary proliferative zones (secondary dentate matrix and external granular layer) are formed. The progenitors in the secondary proliferative zone give rise to small interneurons or granule cells. Production of both neurons in the subpial regions continues into the early postnatal period to form the cerebellar and dentate granule cell layers. These facts suggest that there may be a similar mechanism in early postnatal neurogenesis in the subpial regions of the dentate and cerebellum. Furthermore, the dentate gyrus, unlike the cerebellum, establishes a tertiary proliferative zone (tertiary dentate matrix) in the hilus. During the late postnatal period, the progenitors in this tertiary dentate proliferative zone are gradually confined to the subgranular zone, where neurogenesis persists in adults.

\section{Adult neurogenesis in the mammalian neocortex}

Although adult neurogenesis is a common phenomenon in the non-mammalian neocortex [25], the scale of adult neurogenesis in the neocortex appears to decrease significantly as the phylogeny approaches the human being. In apparent contrast, the limbic area of the telencephalon appears to be a site of continuous generation of neurons. In particular, the medial edge of the mammalian telencephalon, the dentate gyrus, produces granule cells constantly throughout life in humans [26], while the SVZ surrounding the lateral ventricle continuously produces GABAergic granule cells for the olfactory bulb [27].

Granule cells produced in the dentate gyrus are integrated into the circuitry of the hippocampus and save new information, whereas granule cells produced in the SVZ of the lateral ventricle migrate rostrally and are integrated into the olfactory circuitry. In other words, these new neurons are not integrated into the neocortex. However, if a significant number of new neurons is produced in the neocortex and integrated into the neocortical circuit, we cannot deny the possibility that these new neurons ruin the circuits for memory and thought in the human neocortex [28].

Macklis reported that the synchronous apoptotic degeneration of corticothalamic neurons may induce generation of the same number of neurons in the neocortex and may recreate the same circuit as was originally present [29]. In this case, neurogenesis in the neocortex would not disturb the circuit in the neocortex and would be welcomed. Our goal here is to stimulate the interest of many researchers to join the hunt for neuron progenitors in the mouse neocortex.

As the first hunter, Costa et al. sought the source of neuron progenitors using a neurosphere assay method and found a novel source of neuron progenitors outside the two major germinative zones (VZ and SVZ), namely the marginal zone of the neocortex [30]. These neuronal progenitors in the marginal zone of the developing cerebral cortex were notably distinct from those in the VZ and SVZ.

As the second hunter of the neuron progenitors in the adult neocortex, Ohira and colleagues sought NSCs and neuronal progenitor cells (NPCs) in the adult rat neocortex [31]. They induced stress in neocortical cells using global forebrain ischemia. Under these conditions, the vast majority of neocortical neurons were eliminated and the remnant contained more NSCs and NPCs. They then observed NPCs in neocortical layer 1 of adult rats. On injection of a simple retrovirus to visualize these NPCs in layer I, they found that no NPCs or related cells were neurons, but rather had a glia-like appearance. Moreover, none of the NPCs had axons. 


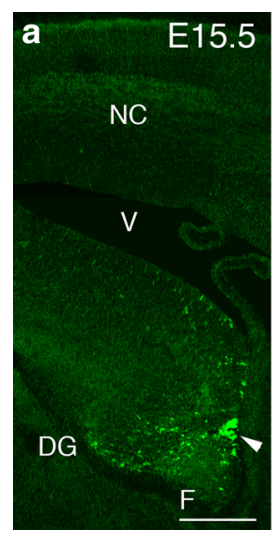

Fig. 2 a Progenitors of granule cells in the developing hippocampus. Gfap-GFP-expressing progenitors (green) arise from the dentate notch (arrowhead) and migrate to the dentate gyrus. $D G$ dentate gyrus, $F$ fimbria, $N C$ neocortex, $V$ ventricle. Bar $200 \mu \mathrm{m}$. b Comparison of neurogenesis among the neocortex, dentate gyrus, and

As the third hunter of the neuron progenitors in the adult neocortex, Nakagomi and colleagues found that neural stem/progenitor cells (NSPCs) reside in many regions of the CNS, including the SVZ of the lateral ventricle, subgranular zone of the hippocampal dentate gyrus, cortex, striatum, and spinal cord [32]. In addition, they demonstrated that the leptomeninges (pia mater and arachnoid membrane) also exhibit NSPC activity in response to ischemia and reported that the NSPCs express NSPC markers, such as nestin, and form neurosphere-like cell clusters with self-renewal activity.

The fourth hunter of neuron progenitors in the adult neocortex is Tamamaki. When the mouse brain was stimulated by electrodes and nestin-immunoreactivity (-IR) in the leptomeninges was examined, neurogenic progenitors were clearly revealed (Fig. 3) [33]. If the electrical stimulation of the brain was continued, nestin-IR was replaced with GAD67-IR or NeuroD6-IR in granule cells. Finally Tuj1-IR is also added to the neuron progenitors in the leptomeninges. When the neocortex was continually stimulated for up to 1 or 2 weeks, neuron progenitors in the leptomeninges migrated into the neocortex and projected dendrites into the cortical plate and axons into the white

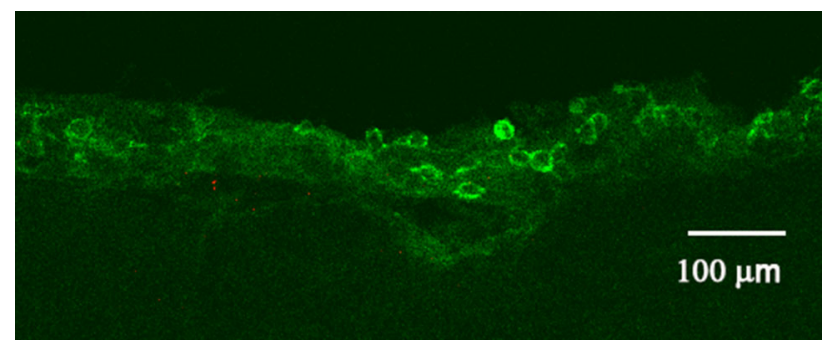

Fig. 3 Piaprogenitors on the outer surface of the pia mater induced by kindling stress. Nestin immunoreactive sites are in green matter. In this case, we consider that the newly generated neocortical neurons would not ruin the circuit in the neocortex.

\section{Understanding the new neurons in the olfactory bulb within the large olfactory neuronal network}

Adult-born olfactory neurons are generated in the SVZ and migrate rostrally to the olfactory bulb $(\mathrm{OB})$, the first relay in olfactory information processing. They then differentiate into granule cells (GCs) or periglomerular cells, which act as interneurons in the OB. In rodents, roughly one percent of total OB GCs are newly generated each day, and more than $60 \%$ of GCs in the adult OB are estimated to be adultborn GCs [3, 34].

Under normal conditions only half of the new GCs succeed in living longer than 1 month after generation, while the other half are eliminated by apoptosis. Initial excess neurogenesis and subsequent elimination commonly occur in both embryonic and adult neurogenesis. Because proper neuronal selection between life and death is crucial to refining the neuronal circuitry throughout the life of the animals, it is important to understand the mechanism of how adult-born neurons are appropriately selected.

First, the selection of adult-born new GCs depends on olfactory sensory experience. Exposure to novel odors increases the survival rate of new GCs, while olfactory sensory deprivation remarkably increases the apoptosis of new GCs $[3,35]$.

Second, the selection of new GCs depends on "brain state." In many cases brain plasticity is influenced by the brain state, most notably by the wake-rest/sleep cycle. In the hippocampus and neocortex, consolidation of spatial 
memory is considered to occur during the rest/sleep period that follows the spatial learning [36]. On the supposition that the selection of new GCs may occur during the time course of olfactory sensory experience and rest/sleep, Yokoyama et al. [37] examined whether the fate decision of new GCs is associated with feeding behavior. Under food restriction, mice initially showed extensive eating behavior following food availability and then showed grooming, resting, and sleeping, which are typical postprandial (after-meal) behaviors. Interestingly, apoptosis of GCs increased approximately two-fold during the postprandial behaviors, whereas perturbation of these postprandial behaviors remarkably suppressed this increase in GC apoptotic elimination. Further, electroencephalogram analysis showed that postprandial slow-wave sleep (SWS) was well correlated with the GC elimination.

So, what mechanisms promote GC elimination during postprandial behaviors, particularly during postprandial SWS? Neurons in the olfactory cortex (OC) repeatedly generated synchronized spike discharges during SWS [38]. Importantly, this synchronized firing of OC neurons generated synchronized top-down synaptic inputs to the OB, which were considered as the candidate signal that promoted GC elimination. In fact, electrical stimulation of the top-down inputs from the $\mathrm{OC}$ to the $\mathrm{OB}$ promoted GC elimination, and pharmacological blockade of the topdown inputs during the postprandial period suppressed GC elimination [39]. These observations demonstrate that topdown inputs during the postprandial period crucially promote GC elimination.

These findings raised the further question of whether enhanced GC elimination occurs only during the postprandial behaviors or during other behavioral states also. Komano-Inoue et al. [40] examined whether fear responses to noxious stimuli induce GC elimination. Mice delivered an electrical foot shock showed startle and fear responses during and immediately after shock delivery. Interestingly, GC elimination was very rapidly enhanced in mice that showed these behavioral responses extensively. Moreover, this enhanced GC elimination after shock delivery was inhibited by the suppression of neuronal activity in the OC.

These results show that GC elimination can be promoted during various behaviors and that top-down inputs from the OC to the OB represent the common signal for inducing GC elimination. One intriguing possibility is that behaviors associated with important life events, such as food eating and experience of noxious stimulation, might promote reorganization of the $\mathrm{OB}$ neuronal circuit, including the enhanced GC elimination. The OC receives synaptic inputs not only from the $\mathrm{OB}$ but also from other nonolfactory brain regions, such as the amygdala and prefrontal cortex. Important life events might recruit wide brain areas for activation and thereby potentiate the activity of the OC and top-down inputs from the $\mathrm{OC}$ to the $\mathrm{OB}$ to reorganize the OB neuronal circuitry.

In conclusion, the life and death of new GCs in the $\mathrm{OB}$ is determined by the interplay between bottom and up olfactory sensory inputs from the periphery and top-down inputs from higher cortical regions (Fig. 4). GCs are presumed to receive these inputs at different synapses in different layers of the OB $[3,41]$. It is important to understand the manner of integration of these synaptic inputs onto a given GC. It is also important to reveal what kind of information is represented by the top-down inputs. Considering that the top-down inputs reflect the activity of higher cortical regions, they might represent the "subjective meaning" of the odor stimuli for the animal, including the valence of the odor and odor-evoked emotional and motivational responses. The fate of new GCs thus needs to be understood with regard to the activity of large neuronal networks.

\section{Relationship between frontal cortical oligodendrocytes and mood}

Major depressive disorder (MDD) is a debilitating mental illness that causes a persistent feeling of sadness, loss of interest, deep despair, and death by suicide. However, the etiology and biological mechanisms of these symptoms are not well understood. Brain imaging studies of patients with MDD have demonstrated abnormalities in gray matter volume, metabolism, and blood flow in the prefrontal cortex and hippocampus [42-44]. Neuropathological studies in these brain regions in MDD have long been of interest in psychiatry. A small number of histological assessments of the prefrontal cortex from patients with MDD revealed abnormalities in the size of neurons and the density of oligodendrocytes [45-47]. However, the stereological techniques often used for the quantification of numbers of cells in brain sections pose a serious methodological challenge to neuropathologists. Using flow cytometry, Hayashi et al. [48] established a novel cellcounting method to quantify the number of nuclei of total cells, neurons, and oligodendrocytes in brain regions of interest from postmortem brains (Fig. 5a, b). The nuclei were obtained by mechanical homogenization of unfixed frozen brain, because the homogenization process breaks the cell membrane and cytoplasm but leaves the nucleus intact. The nuclei in suspension were stained with 7-AAD, NeuN, and Olig2 as markers of DNA (total nuclei), neurons, and oligodendrocytes, respectively, making it possible to quantify the number, size, and fluorescence intensity of the cells with a flow cytometer. Furthermore, the fluorescence intensity of Olig2 immunostaining is stronger in oligodendrocyte precursor cells (OPCs) than in mature 


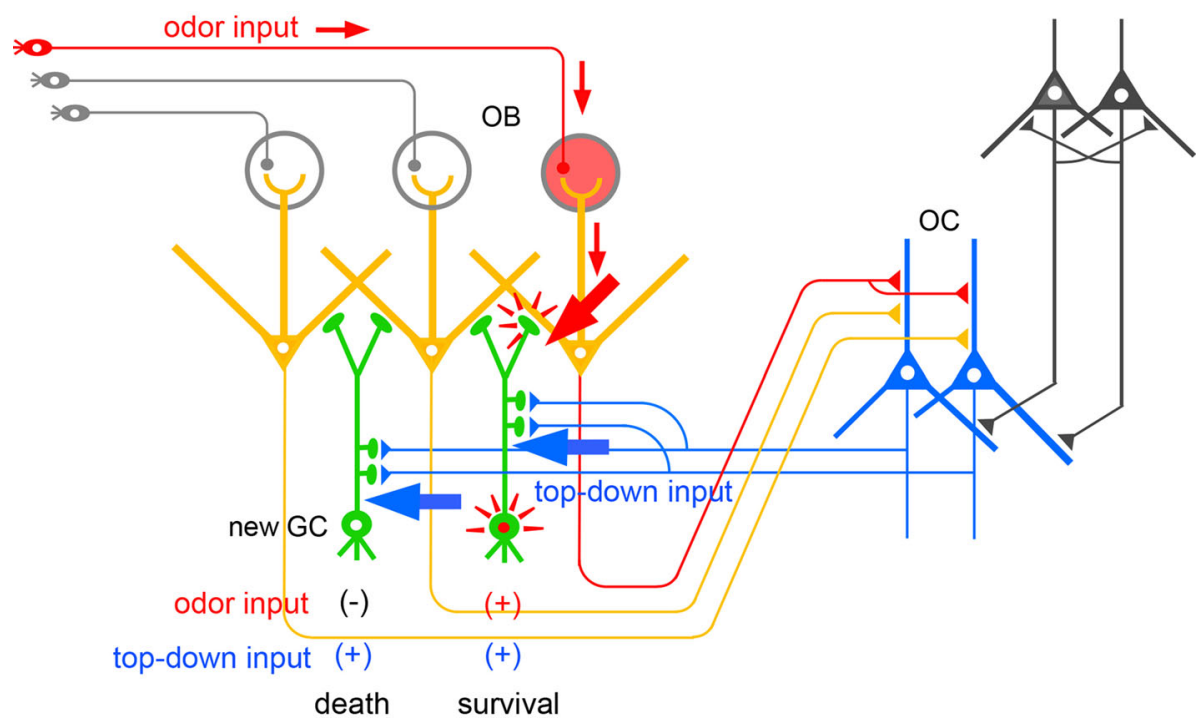

Fig. 4 Activity-dependent selection between survival and death for new GCs in the OB. A model of how synaptic inputs integrate and determine the fate of new GCs. During olfactory experience, some newly generated GCs (green) receive odor input (red arrows for right $G C$ ) while others do not (left $G C$ ). Top-down input from the OC to the $\mathrm{OB}$ (blue arrow), which typically occurs during the sleep state,

oligodendrocytes. The validity and accuracy of this novel cell-counting method have been verified by estimating the number of total and neuronal nuclei from the whole cerebral cortex of rats. This method has been applied to the gray matter of the frontopolar (Brodmann area 10) and inferior temporal (Brodmann area 20) cortices of postmortem brains from MDD patients and healthy controls [49]. In the present report, Hayashi et al. found a reduction in Olig $2^{+}$oligodendrocyte-lineage cells in the gray matter of the frontopolar cortex from MDD patients compared to that from healthy controls (Fig. 5d), but no significant abnormality in the inferior temporal cortex. By measuring the fluorescence intensity, they were able to separate the Olig2 ${ }^{+}$cell population into Olig2 ${ }^{\text {weak }+}$ mature oligodendrocytes and Olig2 $2^{\text {strong }+}$ OPCs (Fig. 5c). Detailed Olig2 ${ }^{+}$ population analysis showed a significant reduction in the densities of both Olig2 $2^{\text {weak+ }}$ and Olig2 $2^{\text {strong+ }}$ nuclei in the frontopolar cortex of MDD patients compared to healthy controls (Fig. 5e, f). These results suggest that the frontopolar cortex, which is considered to be closely associated with the pathogenesis of MDD, processes the oligodendrocyte abnormality and that patients with MDD have a dysfunction in oligodendrocyte development (such as in proliferation and/or maturation) in childhood and/or adolescence.

Continuous neurogenesis in the adult brain is observed in the hippocampus and subependymal zone in rodents as eliminates new GCs that did not receive an odor input (left $G C$ ) and leave new GCs that did receive an odor input to survive (right $G C$ ). OC receives input from other brain regions (gray). Thus, top-down input from the $\mathrm{OC}$ might reflect the activity of various brain regions. This figure was modified from [41]

well as cynomolgus monkeys (Macaca fascicularis) (Fig. $5 \mathrm{~g}, \mathrm{~h}$ ). Volumes of literature report that chronic stress suppresses neurogenesis in rodents and that antidepressants stimulate neurogenesis in the subgranular zone in the hippocampus and the subependymal zone in the lateral ventricles $[50,51]$. Although newly generated oligodendrocytes were observed in the adult hippocampus of a non-human primate [52], oligodendroglial generation from NSCs in the adult brain is controversial [53]. Recently, OPCs that express NG2 chondroitin sulfate were recognized as generators of newly myelinating oligodendrocytes in the adult brain [54]. The proportion of OPCs was reported to constitute about $5 \%$ of total cells in rodent brains [55], which appears comparable to the number of Olig $2^{\text {strong+ }}$ nuclei in the gray matter of the frontopolar cortex of the human brain [49] (Fig. 5i). Because myelination in humans continues from adolescence into the 5th or 6th decade of life (see review [56]), adult oligodendrogenesis in the prefrontal cortex and hippocampus may play a significant role in the pathology of MDD [57, 58]. Thus, it is an urgent issue to understand the molecular mechanisms underlying the generation of OPCs from NSCs, maintenance of OPCs, and production and maturation of oligodendrocytes from OPCs in the adult primate brain and to clarify the causal relationship between the dysfunction of adult OPCs/oligodendrocytes and pathogenesis of MDD. 

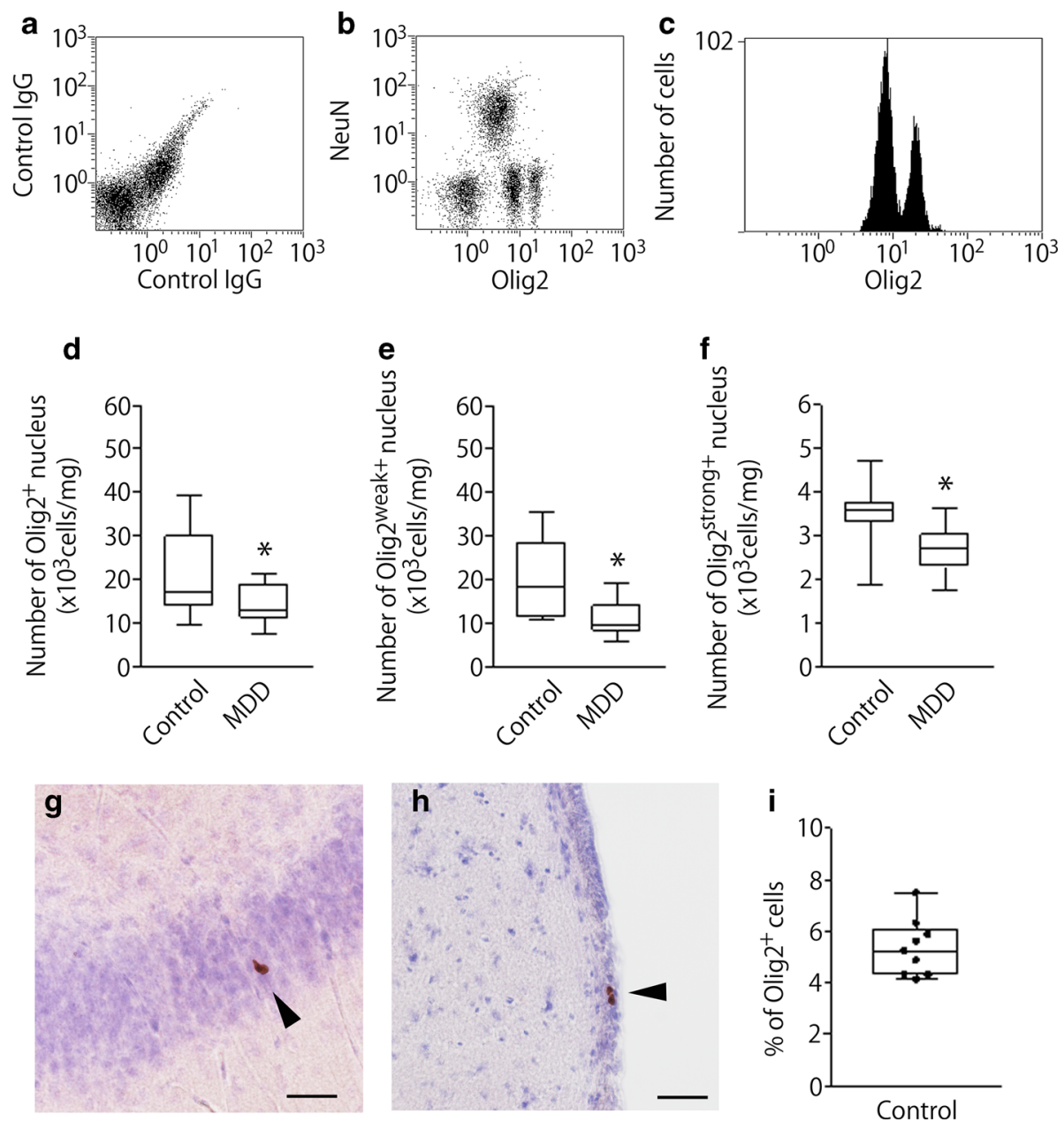

Fig. 5 Quantification of frontopolar cortex from MDD and healthy control. a Negative controls of flow cytometry staining with IgG. b A novel cell counting method can separate nuclei from postmortem brain into neurons $\left(\mathrm{NeuN}^{+}\right)$and oligodendrocytes $\left(\mathrm{Olig} 2^{+}\right)$, astrocytes, and microglia $\left(\mathrm{NeuN}^{-} / \mathrm{Olig} 2^{-}\right)$. c The Olig2 ${ }^{+}$nuclei were further divided into two populations of strongly and weakly Olig2positive nuclei, which are oligodendrocyte precursor cells and oligodendrocytes, respectively. The density of Olig2 ${ }^{+}(\mathbf{d})$, Olig2 ${ }^{\text {weak }+}$ (e), and Olig2 $2^{\text {strong+ }}$ (f) nuclei in $1 \mathrm{mg}$ of gray matter from the

\section{Conclusion and future directions}

These topics indicate that stem cell and neuro/gliogenesis biology is involved in various aspects of neuroscience. This biology encompasses a wide range of animal ages, from the embryo to adulthood; wide brain regions, from the developing neuroepithelium to higher neocortical regions; and various conditions of the brain, including development, maturation, and disease. Collectively, this research field aims to reveal the structural and functional plasticity of the CNS including its genesis, remodeling, and restoration, by incorporating multidisciplinary approaches at the molecular, cellular, circuit, and behavioral levels. frontopolar cortex $\left(\times 10^{3}\right.$ cells $\left./ \mathrm{mg}\right)$. ${ }^{*} p<0.05$ by unpaired $t$ test. Results of immunostaining with Ki-67, a marker of proliferating cells, in the hippocampus (g) and subependymal zone (h) of adult cynomolgus monkeys. Ki-67 ${ }^{+}$cells (brown, arrowhead) are present in both brain regions. Nuclei are counterstained with hematoxylin (purple). Scale bar $40 \mu \mathrm{m}$. i Proportion of Olig $2^{\text {strong+ }}$ nuclei in the frontopolar cortex of healthy controls. A part of this figure was rearranged from [49]

The broad scope of stem cell and neuro/gliogenesis study raises questions about inherent similarities and differences in the properties of NSCs at different animal ages and in different brain regions, as well as in the process of neuro/gliogenesis in the developing, mature, and diseased brain. It appears important to understand both the intrinsic and extrinsic mechanisms of the NSC fate decision, including cell-to-cell interaction between immature cells, between newly generated cells and preexisting cells, and between new healthy cells and old diseased cells.

Further, with regard to functional aspects, how are new neurons and glia incorporated into neural circuits to develop, improve, and restore brain function? To 
understand this, we need to know more about the constitution of functional neural networks and the interactions of the network with new neurons and glia. We also need to determine how neural activity contributes to the appropriate incorporation of new neurons and glia into functional networks. Recent advances in optogenetic and pharmacogenetic approaches will facilitate these studies.

To obtain a comprehensive view, it is crucial to integrate knowledge under different situations and to try to reveal similarities and differences among these situations. The 2015 joint meeting was an ideal opportunity to facilitate the interchange of knowledge among researchers with different backgrounds. We hope that this review will further facilitate this interaction and raise the interest of those who are not directly engaged in this research field. We wish to emphasize that neuro/gliogenesis from NSCs is a drastic process involving significant structural and functional changes in the cells and that integration of newly generated neurons and glia into neural networks is accompanied by significant structural and functional changes in the network. Thus, stem cell and neuro/gliogenesis biology provides an ideal platform for the integration of structural and functional plasticity and will contribute to our understanding of the remarkable plastic potential of the CNS. We also hope that progress in this field will facilitate the interaction of neurobiologists and clinicians and encourage many people by revealing the tremendous plasticity that is buried in our own brain.

\section{References}

1. Taverna E, Götz M, Huttner WB (2014) The cell biology of neurogenesis: toward an understanding of the development and evolution of the neocortex. Annu Rev Cell Dev Biol 30:465-502

2. Altman J (2011) The discovery of adult mammalian neurogenesis. In: Seki $\mathrm{T}$ (ed) Neurogenesis in the adult brain, vol 1 . Springer, Tokyo, pp 3-46

3. Lepousez G, Nissant A, Lledo PM (2015) Adult neurogenesis and the future of the rejuvenating brain circuits. Neuron 86:387-401

4. Sandoe J, Eggan K (2013) Opportunities and challenges of pluripotent stem cell neurodegenerative disease models. Nat Neurosci 16:780-789

5. Kheirbek MA, Klemenhagen KC, Sahay A, Hen R (2012) Neurogenesis and generalization: a new approach to stratify and treat anxiety disorders. Nat Neurosci 15:1613-1620

6. Kriegstein A, Alvarez-Buylla A (2009) The glial nature of embryonic and adult neural stem cells. Annu Rev Neurosci 32:149-184

7. Fishell G, Kriegstein AR (2003) Neurons from radial glia: the consequences of asymmetric inheritance. Curr Opin Neurobiol 13:34-41

8. Imayoshi I, Kageyama R (2014) bHLH factors in self-renewal, multipotency, and fate choice of neural progenitor cells. Neuron 82:9-23

9. Imayoshi I, Kageyama R (2014) Oscillatory control of bHLH factors in neural progenitors. Trends Neurosci 37:531-538
10. Bertrand N, Castro DS, Guillemot F (2002) Proneural genes and the specification of neural cell types. Nat Rev Neurosci 3:517-530

11. Meijer DH, Kane MF, Mehta S, Liu H, Harrington E, Taylor CM, Rowitch DH (2012) Separated at birth? The functional and molecular divergence of OLIG1 and OLIG2. Nat Rev Neurosci 13:819-831

12. Ross SE, Greenberg ME, Stiles CD (2003) Basic helix-loop-helix factors in cortical development. Neuron 39:13-25

13. Wilkinson G, Dennis D, Schuurmans C (2013) Proneural genes in neocortical development. Neuroscience 253:256-273

14. Shimojo H, Ohtsuka T, Kageyama R (2008) Oscillations in notch signaling regulate maintenance of neural progenitors. Neuron 58:52-64

15. Imayoshi I, Isomura A, Harima Y, Kawaguchi K, Kori H, Miyachi H, Kageyama R (2013) Oscillatory control of factors determining multipotency and fate in mouse neural progenitors. Science 342:1203-1208

16. Li G, Pleasure SJ (2014) The development of hippocampal cellular assemblies. Wiley Interdiscip Rev Dev Biol 3:165-177

17. Urban N, Guillemot F (2014) Neurogenesis in the embryonic and adult brain: same regulators, different roles. Front Cell Neurosci $8: 396$

18. Seki $\mathrm{T}$ (2011) From embryonic to adult neurogenesis in the dentate gyrus. In: Seki $\mathrm{T}$ et al (eds) Neurogenesis in the adult brain, vol 1. Springer, Tokyo, pp 193-216

19. Seki T, Sato T, Toda K, Osumi N, Imura T, Shioda S (2014) Distinctive population of Gfap-expressing neural progenitors arising around the dentate notch migrate and form the granule cell layer in the developing hippocampus. J Comp Neurol 522:261-283

20. Li G, Kataoka H, Coughlin SR, Pleasure SJ (2009) Identification of a transient subpial neurogenic zone in the developing dentate gyrus and its regulation by $\mathrm{Cxcl12}$ and reelin signaling. Development 136:327-335

21. Rickmann M, Amaral DG, Cowan WM (1987) Organization of radial glial cells during the development of the rat dentate gyrus. J Comp Neurol 264:449-479

22. Altman J, Bayer SA (1990) Mosaic organization of the hippocampal neuroepithelium and the multiple germinal sources of dentate granule cells. J Comp Neurol 301:325-342

23. Sievers J, Hartmann D, Pehlemann FW, Berry M (1992) Development of astroglial cells in the proliferative matrices, the granule cell layer, and the hippocampal fissure of the hamster dentate gyrus. J Comp Neurol 320:1-32

24. Yuasa S (2001) Development of astrocytes in the mouse hippocampus as tracked by tenascin-C gene expression. Arch Histol Cytol 64:149-158

25. Kaslin J, Ganz J, Brand M (2008) Proliferation, neurogenesis and regeneration in the non-mammalian vertebrate brain. Philos Trans R Soc B 363:101-122

26. Bayer SA, Altman J (1991) Neocortical development. Raven Press, Johannesburg

27. Doetsch F, Caillé I, Lim DA, García-Verdugo JM, AlvarezBuylla A (1999) Subventricular zone astrocytes are neural stem cells in the adult mammalian brain. Cell 97:703-716

28. Rakic P (2004) Neuroscience: immigration denied. Nature 427:685-686

29. Magavi SS, Leavitt BR, Macklis JD (2000) Induction of neurogenesis in the neocortex of adult mice. Nature 405:951-955

30. Costa MR, Kessaris N, Richardson WD, Gotz M, Hedin-Pereira C (2007) The marginal zone/layer I as a novel niche for neurogenesis and gliogenesis in developing cerebral cortex. J Neurosci 27:11376-11388

31. Ohira K, Furuta T, Hioki H, Nakamura KC, Kuramoto E, Tanaka Y, Funatsu N, Shimizu K, Oishi T, Hayashi M, Miyakawa T, 
Kaneko T, Nakamura S (2010) Ischemia-induced neurogenesis of neocortical layer 1 progenitor cells. Nat Neurosci 13:173-179

32. Nakagomi T, Molnar Z, Taguchi A, Nakano-Doi A, Lu S, Kasahara Y, Nakagomi N, Matsuyama T (2012) Leptomeningealderived doublecortin-expressing cells in poststroke brain. Stem Cells Dev 21:2350-2354

33. Ninomiya S, Esumi S, Ohta K, Fukuda T, Ito T, Imayoshi I, Kageyama R, Ikeda T, Itohara S, Tamamaki N (2013) Amygdala kindling induces nestin expression in the leptomeninges of the neocortex. Neurosci Res 75:121-129

34. Imayoshi I, Sakamoto M, Ohtsuka T, Takao K, Miyakawa T, Yamaguchi M, Mori K, Ikeda T, Itohara S, Kageyama R (2008) Roles of continuous neurogenesis in the structural and functional integrity of the adult forebrain. Nat Neurosci 11:1153-1161

35. Yamaguchi M, Mori K (2005) Critical period for sensory experience-dependent survival of newly generated granule cells in the adult mouse olfactory bulb. Proc Natl Acad Sci USA 102:9697-9702

36. Diekelmann S, Born J (2010) The memory function of sleep. Nat Rev Neurosci 11:114-126

37. Yokoyama TK, Mochimaru D, Murata K, Manabe H, Kobayakawa K, Kobayakawa R, Sakano H, Mori K, Yamaguchi M (2011) Elimination of adult-born neurons in the olfactory bulb is promoted during the postprandial period. Neuron 71:883-897

38. Manabe H, Kusumoto-Yoshida I, Ota M, Mori K (2011) Olfactory cortex generates synchronized top-down inputs to the olfactory bulb during slow-wave sleep. J Neurosci 31:8123-8133

39. Komano-Inoue S, Manabe H, Ota M, Kusumoto-Yoshida I, Yokoyama TK, Mori K, Yamaguchi M (2014) Top-down inputs from the olfactory cortex in the postprandial period promote elimination of granule cells in the olfactory bulb. Eur J Neurosci 40:2724-2733

40. Komano-Inoue S, Murata K, Mori K, Yamaguchi M (2015) Rapid induction of granule cell elimination in the olfactory bulb by noxious stimulation in mice. Neurosci Lett 598:6-11

41. Yamaguchi M, Manabe H, Murata K, Mori K (2013) Reorganization of neuronal circuits of the central olfactory system during postprandial sleep. Front Neural Circuits 7:132

42. Drevets WC, Bogers W, Raichle ME (2002) Functional anatomical correlates of antidepressant drug treatment assessed using PET measures of regional glucose metabolism. Eur Neuropsychopharmacol 12:527-544

43. Frodl T, Meisenzahl EM, Zetzsche T, Born C, Groll C, Jager M, Leinsinger G, Bottlender R, Hahn K, Moller HJ (2002) Hippocampal changes in patients with a first episode of major depression. Am J Psychiatry 159:1112-1118

44. Chang CC, Yu SC, McQuoid DR, Messer DF, Taylor WD, Singh K, Boyd BD, Krishnan RR, MacFall JR, Steffens DC, Payne ME (2011) Reduction of dorsolateral prefrontal cortex gray matter in late-life depression. Psychiatry Res 193:1-6

45. Cotter D, Mackay D, Chana G, Beasley C, Landau S, Everall IP (2002) Reduced neuronal size and glial cell density in area 9 of the dorsolateral prefrontal cortex in subjects with major depressive disorder. Cereb Cortex 12:386-394

46. Uranova NA, Vostrikov VM, Orlovskaya DD, Rachmanova VI (2004) Oligodendroglial density in the prefrontal cortex in schizophrenia and mood disorders: a study from the Stanley Neuropathology Consortium. Schizophr Res 67:269-275

47. Vostricov V, Uranova N (2011) Age-related increase in the number of oligodendrocytes is dysregulated in schizophrenita and mood disorders. Schizophr Res Treat 2011:174689

48. Hayashi Y, Kikuchi NN, Hisanaga SI, Tatebayashi Y (2011) A novel, rapid, cell-counting method for unfixed frozen brains comprehensively quantifies at least four neural cell populations. Mol Psychiatry 16:1155

49. Hayashi Y, Kikuchi NN, Yu X, Ishimoto K, Hisanaga SI, Tatebayashi Y (2011) A novel, rapid, quantitative cell-counting method reveals oligodendroglial reduction in the frontopolar cortex in major depressive disorder. Mol Psychiatry 16:1156-1158

50. Malberg J, Eisch AJ, Nestler EJ, Duman RS (2000) Chronic antidepressant treatment increases neurogenesis in adult rat hippocampus. J Neurosci 20:9104-9110

51. Hitoshi S, Maruta N, Higashi M, Kumer A, Kato N, Ikenaka K (2007) Antidepressant drugs reverse the loss of adult neural stem cells following chronic stress. J Neurosci Res 85:3574-3585

52. Kornack DR, Rakic P (1999) Continuation of neurogenesis in the hippocampus of adult macaque monkey. Proc Natl Acad Sci USA 96:5768-5773

53. Bonaguidi MA, Wheeler MA, Shapiro JS, Stadel RP, Sun GJ, Ming GL, Song H (2011) In vivo clonal analysis reveals selfrenewing and multipotent adult neural stem cell characteristics. Cell 145:1142-1155

54. Rivers LE, Young KM, Rizzi M, Jamen F, Psachoulia K, Wade A, Kessaris N, Richardson W (2008) PDGFRA/NG2 glia generate myelinating oligodendrocytes and piriform projection neurons in adult mice. Nat Neurosci 11:1392-1401

55. Livine JM, Raynolds R, Fawcett JW (2001) The oligodendrocyte precursor cell in health and disease. Trends Neurosci 24:39-47

56. Kikuchi NN, Hayashi Y, Yu XJ, Tatebayashi Y (2013) Depression and alzheimer's disease: novel postmortem barin studies reveal a possible common mechanism. J Alzheimers Dis 37:611-621

57. Elsayed M, Banasr M, Duric V, Fournier NM, Licznerski P, Duman R (2012) Antidepressant effects of fibroblast growth factor-2 in behavioral and cellular models of depression. Biol Psychiatry 72:258-265

58. Chetty S, Friedman AR, Lahn KT, Kirby ED, Mirescu C, Guo F, Krupik D, Nicholas A, Geraghty AC, Krishnamurthy A, Tsai MK, Covarrubias D, Wong AT, Francis DD, Sapolskey RM, Palmer TD, Pleasure D, Kaufe D (2014) Stress and glucocorticoids promote oligodendrogenesis in the adult hippocampus. Mol Psychiatry 19:1275-1283 